\title{
Damping of coupled phonon-plasmon modes
}

\author{
L. A. Falkovsky \\ Landau Institute for Theoretical Physics, 117337 Moscow, Russia
}

\begin{abstract}
The effect of free carriers on dispersion and damping of coupled phonon-plasmon modes is considered in the long-wave approximation. The electron and phonon scattering rate as well as Landau damping are taken into account.
\end{abstract}

*Electronic address: falk@itp.ac.ru 


\section{INTRODUCTION}

After the pioneering paper by Migdal [1], the effect of electron-phonon interactions on the phonon dispersion has been permanently debated. The problem was how to explain that the many-body approach based on the Fröhlich Hamiltonian gives a strong phonon renormalization. For instance, the sound velocity is renormalized on the order of phonon-electron coupling $\lambda$ which has the value of unity. It follows that the phonon-electron system can be unstable. The phonon softening caused by the electron-phonon interaction have been discussed in many papers. All these results contradict to the Born-Oppenheimer conception [2] according to which the light electrons must follow the slow lattice vibrations. Therefore, the phonon

renormalization should involve the small parameter of nonadiabacity $\sqrt{m / M}$, where $m$ and $M$ are the electron and ion masses correspondingly.

The discrepancy was resolved by Brovman and Kagan [3]. They demonstrated the shortcomings of the Fröhlich model. Employing the adiabatic approximation, they found that there are two terms in the second order perturbation theory, which compensate each other making a result small by the nonadiabatic parameter.

But recently, Alexandrov and Schrieffer [4] again obtained the strong phonon renormalization and predicted an extremely large dispersion of optical phonons (on order of the Fermi velocity) because of coupling to electrons. The large phonon dispersion is a typical result of the theory [5] used the Frölich Hamiltonian. Reizer [6] stressed the importance of screening effects accompanying the longitudinal optical modes, but the nonphysical renormalization remained in his results. For the first time, the screening of the Coulomb field in optical vibrations was treated in the paper [7].

Only the phonon frequencies were calculated in the works [4], [6], [7], with no results available for the attenuation of optical phonons. The electron collisions with each other, with defects and phonons were ignored. Beside the electron collision rate $\gamma$, the natural phonon width $\Gamma^{\text {nat }}$ was also disregarded. The natural phonon 
width is caused by the anharmonicity processes of the phonon decay into two (or more) phonons. Notice, that the collision processes determine the conductivity and the dielectric permittivity, i. e. electrodynamics of the electron-phonon system. In the optic range, the collision rates $\gamma, \Gamma^{\text {nat }} \sim \sqrt{m / M} \omega_{\mathrm{O}}$ are small compared with a typical phonon frequency $\omega_{\mathrm{O}}$ and they give the widths of the plasmon and phonon resonances. Experimental studies of this resonances provide informations about isotopic compositions and quality of semiconductor materials.

Using the Boltzmann equation for electrons and the equation of motion for phonons we calculated [9] the frequency shift and the width of optical phonons in metals. We take into account all the mentioned features: the electron-phonon interaction of different forms, the Coulomb screening, and the collision rates of electrons and phonons. We find that dispersion and damping of the longitudinal modes can be correctly described if we neglect the direct electron-phonon interaction $\lambda$ and keep only the screening and the collision rates $\gamma, \Gamma^{\text {nat }}$. Emphasize, that in the semiclassical approximation, when the phonon momentum transferred to electrons is small compared with the electron momentum, the method of the Boltzmann equation is completely equivalent to the diagram technique. The corresponding equations may be formulated as equations for the electron and phonon self-energies. But in any case, we must properly incorporate adiabatic approximation and screening.

Experimentally, it is convenient to investigate the free-carrier effect on phonon modes, varying the carrier concentration, i. e. for doped semiconductors or superconductors (see, for example, the recent work [10], carried out on the HTcS compound $\mathrm{Nd}_{1.86} \mathrm{Ce}_{0.14} \mathrm{CuO}_{4+\delta}$ using IXS). Therefore, in the present paper, we extend the results of the paper [9] to the case of small free-carrier concentrations when the electron plasma frequency $\omega_{e p}$ can be of the order of the phonon frequency $\omega_{O}$, and the coupled phonon-plasmon modes can exist. We consider the screening as well as the collision effects $\gamma$ and $\Gamma^{\text {nat }}$, focusing on the width of the phonon-plasmon modes. We simplify essentially the problem ignoring the direct electron-phonon interaction (terms with $\lambda$ ) and assuming that, first, the electron system is degenerate and, sec- 
ond, the momentum transfer $k$ is small compared with the Fermi momentum $p_{F}$.

\section{ASYMPTOTIC EXPRESSIONS FOR THE DIELECTRIC FUNCTION}

Let us find the limiting expressions of the dielectric function

$$
\varepsilon(k, \omega)=\varepsilon_{\infty} \frac{\omega^{2}-\omega_{\mathrm{LO}}^{2}+i \Gamma^{\mathrm{nat}}}{\omega^{2}-\omega_{\mathrm{TO}}^{2}+i \Gamma^{\text {nat }}}-\frac{4 \pi e^{2} \nu_{0}\left\langle v_{z} / \Delta_{p}(k)\right\rangle}{k\left(1-i\left\langle\gamma / \Delta_{p}(k)\right\rangle\right)} .
$$

Here the ion contribution (first term) is assumed as dispersionless, while we are interested in small wave vectors in comparison with the Brillouin-zone size. This term has the pole and the zero at the frequencies of the transverse and longitudinal phonons correspondingly. The natural phonon width $i \Gamma^{\text {nat }} / 2$ is added to $\omega$; the high-frequency ion permittivity is denoted $\varepsilon_{\infty}$.

The second term in Eq. (田) is the electron contribution into permittivity for $k \ll p_{F}$, written [9] with the help of the Boltzmann equation in approximation of the collision rate $\gamma$; the notation

$$
\Delta_{p}(k)=\omega-\mathbf{k v}+i \gamma
$$

is introduced. The angular brackets

$$
\langle\ldots\rangle=\frac{1}{\nu_{0}} \int(\ldots) \frac{2 d S_{F}}{v(2 \pi)^{3}} .
$$

denote averaging over the Fermi surface with the density of states $\nu_{0}$. For the isotropic case, the density of states on the Fermi surface $\nu_{0}=m^{*} p_{F} / \pi^{2}$ and $m^{*}$ is the effective electron mass.

Let us rewrite the electron contribution

$$
\varepsilon_{e}(k, \omega)=\varepsilon_{\infty} \frac{k_{0}^{2}}{k^{2}}\left[1-\frac{\left\langle\omega / \Delta_{p}(k)\right\rangle}{1-i\left\langle\gamma / \Delta_{p}(k)\right\rangle}\right],
$$

where the Thomas-Fermi parameter $k_{0}^{2}=4 \pi e^{2} \nu_{0} / \varepsilon_{\infty}$.

For the isotropic Fermi surface we can carry out the integration

$$
\left\langle 1 / \Delta_{p}(k)\right\rangle=\frac{1}{2 k v_{F}} \ln \frac{\omega+i \gamma+k v_{F}}{\omega+i \gamma-k v_{F}} .
$$


Separating the imaginary and real parts we have

$$
\left\langle 1 / \Delta_{p}(k)\right\rangle=\frac{1}{2 k v_{F}}\left[\frac{1}{2} \ln \frac{\left(\omega+k v_{F}\right)^{2}+\gamma^{2}}{\left(\omega-k v_{F}\right)^{2}+\gamma^{2}}+i \arctan \frac{\omega-k v_{F}}{\gamma}-i \arctan \frac{\omega+k v_{F}}{\gamma}\right] .
$$

The imaginary part known as the Landau damping is pronounced at $k v_{F}>\mid \omega+$ $i \gamma \mid:$

$$
\varepsilon_{e}(k, \omega)=\varepsilon_{\infty}\left(k_{0} / k\right)^{2}\left(1+i \pi \omega / 2 k v_{F}\right), \quad k v_{F}>>|\omega+i \gamma|
$$

In the range $\gamma<\omega-k v_{F}<<k v_{F}$, we have

$$
\varepsilon_{e}(k, \omega)=\varepsilon_{\infty} \frac{k_{0}^{2}}{k^{2}}\left\{1-\frac{\omega}{2 k v_{F}}\left[\frac{1}{2} \ln \frac{4 k^{2} v_{F}^{2}}{\left(\omega-k v_{F}\right)^{2}+\gamma^{2}}-i \frac{\gamma}{\omega-k v_{F}}\right]\right\} .
$$

For the small $k v_{F}<<|\omega+i \gamma|$, the expansion in $k v_{F} /(\omega+i \gamma)$ gives

$$
\varepsilon_{e}(k, \omega)=\varepsilon_{\infty}\left\{1-\frac{\omega_{p e}^{2}}{\omega(\omega+i \gamma)}\left[1+\frac{3}{5}\left(\frac{k v_{F}}{\omega+i \gamma}\right)^{2}\right]\right\} .
$$

Here the $k$-independent term represents the Drude conductivity. The electron plasma frequency is given by the integral over the Fermi surface

$$
\omega_{p e}^{2}=\frac{e^{2}}{3 \pi^{2} \varepsilon_{\infty}} \int v d S_{F} .
$$

The limiting expressions (41)-(有) are also valid for the arbitrary Fermi surface, but the constant $v_{F}$ has different values. In Eq. (4), this is an average velocity on the belt $\mathbf{v} \perp \mathbf{k}$, the velocity in the limiting point $\mathbf{v} \| \mathbf{k}$ in Eq. (5) , and the squared velocity averaged over the whole Fermi surface in Eq. (6). We do not pay attention the cases when the Fermi surface have flat or cylindrical pieces.

\section{FREQUENCY AND DAMPING OF PHONON-PLASMON MODES}

The frequencies of the longitudinal phonon-plasmon modes are determined by the equation $\varepsilon(k, \omega)=0$. In the absence of the electron and phonon collisions $\left(\gamma=\Gamma^{\text {nat }}=0\right)$, we obtain with the help of Eqs. (1) and (6) the biquadratic equation. It gives the frequencies of the coupled phonon-plasmon modes at $k=0$ :

$$
\omega_{ \pm}^{2}=\frac{1}{2}\left(\omega_{p e}^{2}+\omega_{\mathrm{LO}}^{2}\right) \pm \frac{1}{2}\left[\left(\omega_{p e}^{2}+\omega_{\mathrm{LO}}^{2}\right)^{2}-4 \omega_{p e}^{2} \omega_{\mathrm{TO}}^{2}\right]^{1 / 2} .
$$


These frequencies (related to the $\omega_{\mathrm{TO}}$ ) are shown in Fig. 1 (left panel) as functions of the electron concentration, namely, $\omega_{p e} / \omega_{\mathrm{TO}}$. The upper dashed line begins at $\omega_{\mathrm{LO}}$ and tends to the electron plasma frequency $\omega_{p e}$. The lower frequency (solid line) starts as $\omega_{p e} \omega_{\mathrm{TO}} / \omega_{\mathrm{LO}}$ and then approaches $\omega_{\mathrm{TO}}$. In other words, observing in the optic range the longitudinal phonon mode and adding electrons, we see a transition of the longitudinal phonon frequency from $\omega_{\mathrm{LO}}$ to $\omega_{\mathrm{TO}}$. This is a result of the Coulomb screening.

Since both the collision rates $\gamma$ and $\Gamma^{\text {nat }}$ in the optic range are small in comparison with $\omega_{\mathrm{O}}$, the damping of the phonon-plasmon modes can be added to their frequencies $\omega=\omega_{ \pm}-i \Gamma_{ \pm} / 2$. Using Eqs. (回) and (7) we find at $k=0$ :

$$
\begin{aligned}
& \Gamma_{+}=\left[\gamma\left(\omega_{+}^{2}-\omega_{\mathrm{LO}}^{2}\right)+\Gamma^{\mathrm{nat}}\left(\omega_{+}^{2}-\omega_{p e}^{2}\right)\right] /\left(\omega_{+}^{2}-\omega_{-}^{2}\right), \\
& \Gamma_{-}=\left[\gamma\left(\omega_{-}^{2}-\omega_{\mathrm{LO}}^{2}\right)+\Gamma^{\mathrm{nat}}\left(\omega_{-}^{2}-\omega_{p e}^{2}\right)\right] /\left(\omega_{-}^{2}-\omega_{+}^{2}\right) .
\end{aligned}
$$

The behavior of damping as a function of the electron concentration is shown in Fig. 1 (right panel) for the ratio $\gamma / \Gamma^{\text {nat }}=3$. We see that the concentration dependence of widths corresponds with the type of modes: for the low electron concentration, the solid line represents mainly the electron plasma mode and the dashed line is associated with the longitudinal phonon. The character of modes reverses at the higher electron concentrations.

Now let us consider the dispersion of the coupled phonon-plasmon modes. When the wave vector $k$ increases, the upper mode (see Fig. 2 and Eq. (5)) approaches the asymptote $\omega=k v_{F}$ :

$$
\omega_{+}=k v_{F}\left\{1+2 \exp \left[-2-\frac{k^{2}\left(k^{2} v_{F}^{2}-\omega_{\mathrm{LO}}^{2}\right)}{k_{0}^{2}\left(k^{2} v_{F}^{2}-\omega_{\mathrm{TO}}^{2}\right)}\right]\right\}-i \gamma .
$$

The lower mode drops into the domain $k v_{F}>\omega$ (dashed curve), where the Landau damping appears:

$$
\omega_{-}^{2}=\frac{k^{2} \omega_{\mathrm{LO}}^{2}+k_{0}^{2} \omega_{\mathrm{TO}}^{2}}{k^{2}+k_{0}^{2}}-i \frac{\pi \omega k k_{0}^{2} \omega_{p i}^{2}}{2 v_{F}\left(k^{2}+k_{0}^{2}\right)^{2}} .
$$

Here the ion plasma frequency $\omega_{p i}^{2}=\omega_{\mathrm{LO}}^{2}-\omega_{\mathrm{TO}}^{2}$. In the imaginary part, we should substitute instead of $\omega$ the frequency $\omega_{-}$defined by the real part. Let us emphasize, 
that the large dispersion arises under the conditions $k_{0} v_{F} \gg \omega$ and $k_{0}<p_{F}$. Then, at $k \simeq k_{0}$, the frequency changes from $\omega_{\mathrm{TO}}$ to $\omega_{\mathrm{LO}}$. Again this is the effect of screening.

In the general case, the frequency and the damping of phonon-plasmon modes can be found in the numerical solution of the equation $\varepsilon(k, \omega)=0$. But of particular interest is the function $-\operatorname{Im} 1 / \varepsilon(k, \omega)$, since it gives the intensity of the inelastic Raman or X-ray scattering, where $\omega$ and $\mathbf{k}$ have the sense of frequency and momentum transfers, respectively. The plots of the intensity, obtained with the help of Eqs. (11) - (3) are shown in Figs. $3-5$ for the cases of the large electron density $\left(\omega_{p e}>\omega_{\mathrm{TO}}\right)$. The peak at $\omega / \omega_{\mathrm{TO}}=2.6 \div 2.7$ corresponds to the plasmon excitation; notice, that $\omega_{p e}=k_{0} v_{F} / \sqrt{3}$ for the quadratic electron dispersion.

In the domain $\omega / \omega_{\mathrm{TO}}<1$, there is a phonon peak (see left panel of Fig. 1 and Eq. (7) ). Because of the screening, its frequency is smaller than $\omega_{\mathrm{LO}}$. For the small $k$ (Fig. 3), the intensity of the phonon peak is 50 times less than the plasmon-peak intensity. For the larger $k$ (see Fig. 4), the broad continuum $\omega<k v_{F}$ appears on the low-frequency side of the phonon peak. Here the dielectric constant $\varepsilon(k, \omega)$ has a noticeable imaginary part (14) arising from the electron-hole excitations. The intensity of the phonon peak decreases, its line shape becomes asymmetric similar to the Fano resonance. Finally, the phonon peak is spread, being immersed in the electron continuum (see Fig. 5 and Eq. (8)).

For the relative low electron density, $\omega_{p e}<\omega_{\text {TO }}$, the intensities are shown in Figs. $6-8$. Now the phonon peak is approximately at $\omega / \omega_{\mathrm{TO}}=1.5$. That yields $\omega=\omega_{\mathrm{LO}}$, since we set $\omega_{\mathrm{LO}} / \omega_{\mathrm{TO}}=\sqrt{2}$. There are also the plasmon peak and the electron-hole continuum. In Fig. 6 the plasmon peak is broader than the phonon peak, since we put $\gamma / \Gamma^{\text {nat }}=3$. The plasmon peak becomes more broader in Fig. 7 because of the neighboring electron continuum at $\omega<k v_{F}$. It disappears completely in Fig. 8. 


\section{CONCLUSION}

We have shown that the width of the longitudinal phonon-plasmon modes increases with increasing of the free-carrier concentration because their frequency approaches the region $k v_{F}>\omega$, where the electron-hole excitations exist. The detailed experimental studies could help to clarify the comparative role of the different mechanisms of the phonon shift and damping in the metallic and doped semiconducting materials under the influence of the electron concentration.

The work was partially supported by the RFBR (project 01-02-16211).

[1] A. B. Migdal, Zh. Eksp. Teor. Fiz. 34, 1438 (1958) [Sov. Phys. JETP 7, 996 (1958)].

[2] M. Born and Kung Huang, Dynamical Theory of Crystal Lattices (Oxford University Press, New York, 1954).

[3] E. G. Brovman and Yu. Kagan, Zh. Eksp. Teor. Fiz. 52, 557 (1967) [Sov. Phys. JETP 25, 365 (1967)].

[4] A. S. Alexandrov and J. R. Schrieffer, Phys. Rev. B 56, 13731 (1997).

[5] A. A. Abrikosov, L. P. Gor'kov, I. Ye. Dzyaloshinskii, Methods of Quantum Field Theory in Statistical Physics, (Prentice-Hall, Englewood Cliffs, NJ, 1963).

[6] M. Reizer, Phys. Rev. B 61, 40 (2000).

[7] V. L. Gurevich, A. I. Larkin, and Yu. A. Firsov, Fiz. Tv. Tela 4, 185 (1962).

[8] V. M. Kontorovich, Usp. Fiz. Nauk 142, 265 (1984) [Sov. Phys. Uspekhi 27, 134 (1984)].

[9] L. A. Falkovsky, Zh. Eksp. Teor. Fiz. 122, 411 (2002) [Sov. Phys. JETP 95, No 2(8) (2002)].

[10] M. d'Adusto, P. K. Mang, P. Giura, A. Shukla, P. Chigna, A. Mirone, M. Braden, M. Greven, M. Krish, and F. Sette, cond-mat/0201501 (2002). 


\section{Figure captions:}

Fig. 1. Frequencies (in units of $\omega_{\mathrm{TO}}$, left panel) and widths (in units of $\Gamma^{\text {nat }}$, right panel) of the phonon-plasmon modes at $k=0$ in dependence of the free carrier concentration, namely, of the electron plasma frequency (in units of $\omega_{\mathrm{TO}}$ ). We set the ratio of the $\mathrm{LO}$ and $\mathrm{TO}$ frequencies in the absence of the free carriers $\omega_{\mathrm{LO}} / \omega_{\mathrm{TO}}=\sqrt{2}$ and the ratio of the electron and phonon damping $\gamma / \Gamma^{\mathrm{nat}}=3$.

Fig. 2. The dispersion of phonon-plasmon modes for the metallic $\left(\omega_{p e}>\omega_{\mathrm{TO}}\right.$, left panel) and semiconducting ( $\omega_{p e}<\omega_{\mathrm{TO}}$, right panel) carrier concentration. The dashed straight lines separate the domain $k v_{F}>\omega$, where the Landau damping exists; the dashed curves represent the strongly damped modes.

Fig. $3-8$. The inelastic light scattering intensity $-\operatorname{Im} 1 / \varepsilon(k, \omega)$ as a function of the frequency transfer $\omega$ for the momentum transfer $k$ and the Thomas-Fermi parameter $k_{0}$ (in the units of $\omega_{\mathrm{TO}} / v_{F}$ ) shown in the figures. We set $\omega_{\mathrm{LO}} / \omega_{\mathrm{TO}}=\sqrt{2}$, $\Gamma^{\text {nat }} / \omega_{\mathrm{TO}}=10^{-2}, \gamma / \Gamma^{\text {nat }}=3$. 

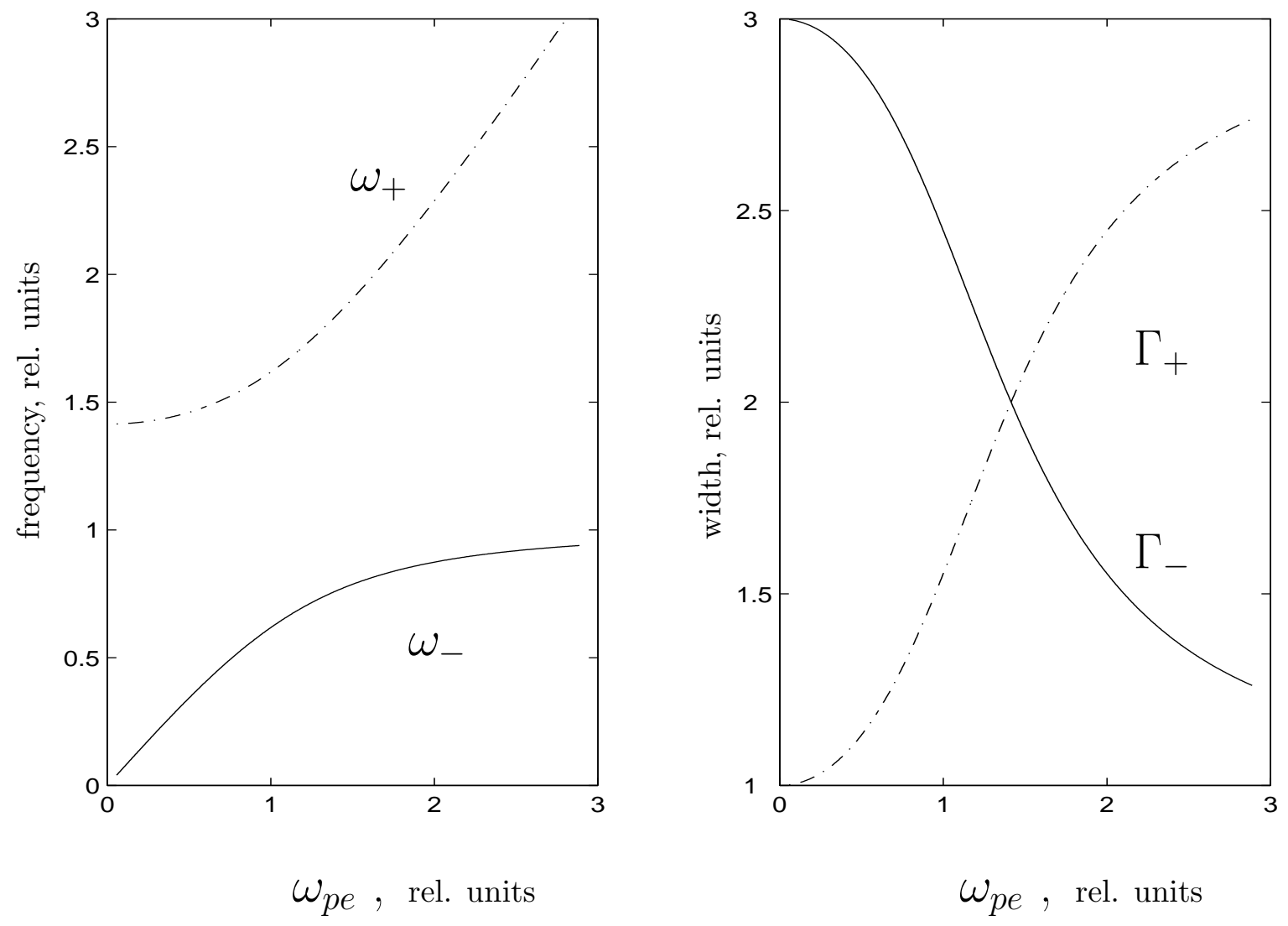

FIG. 1: 


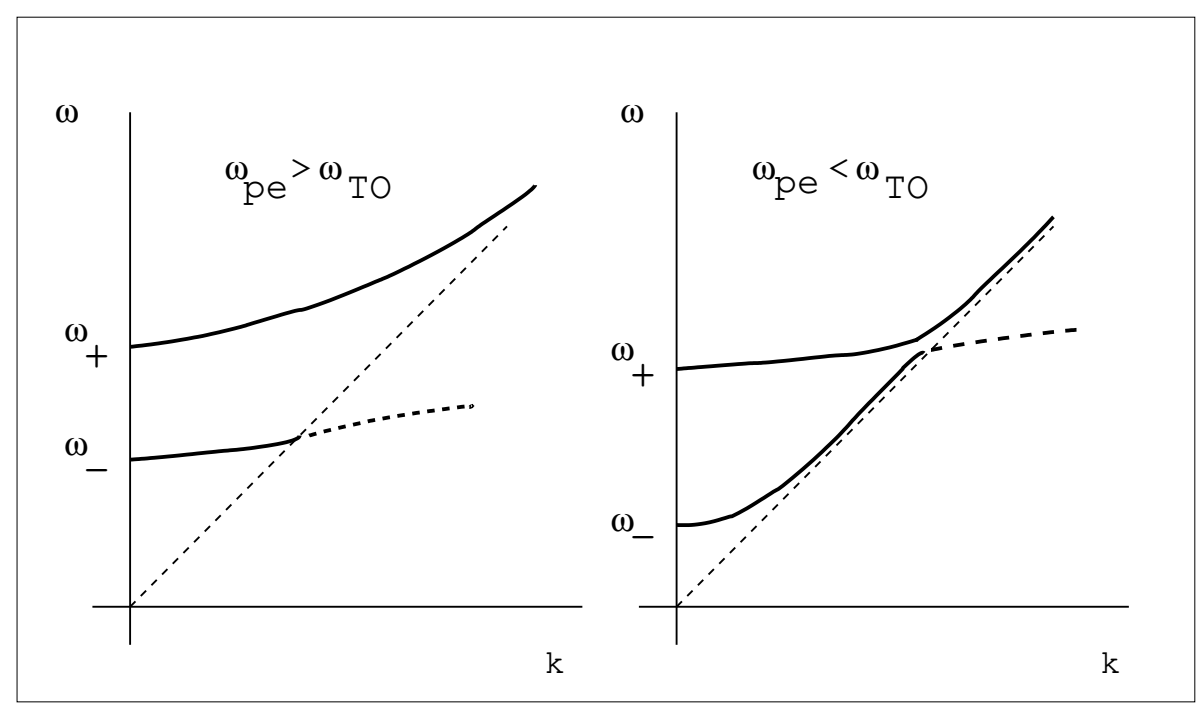

FIG. 2: 


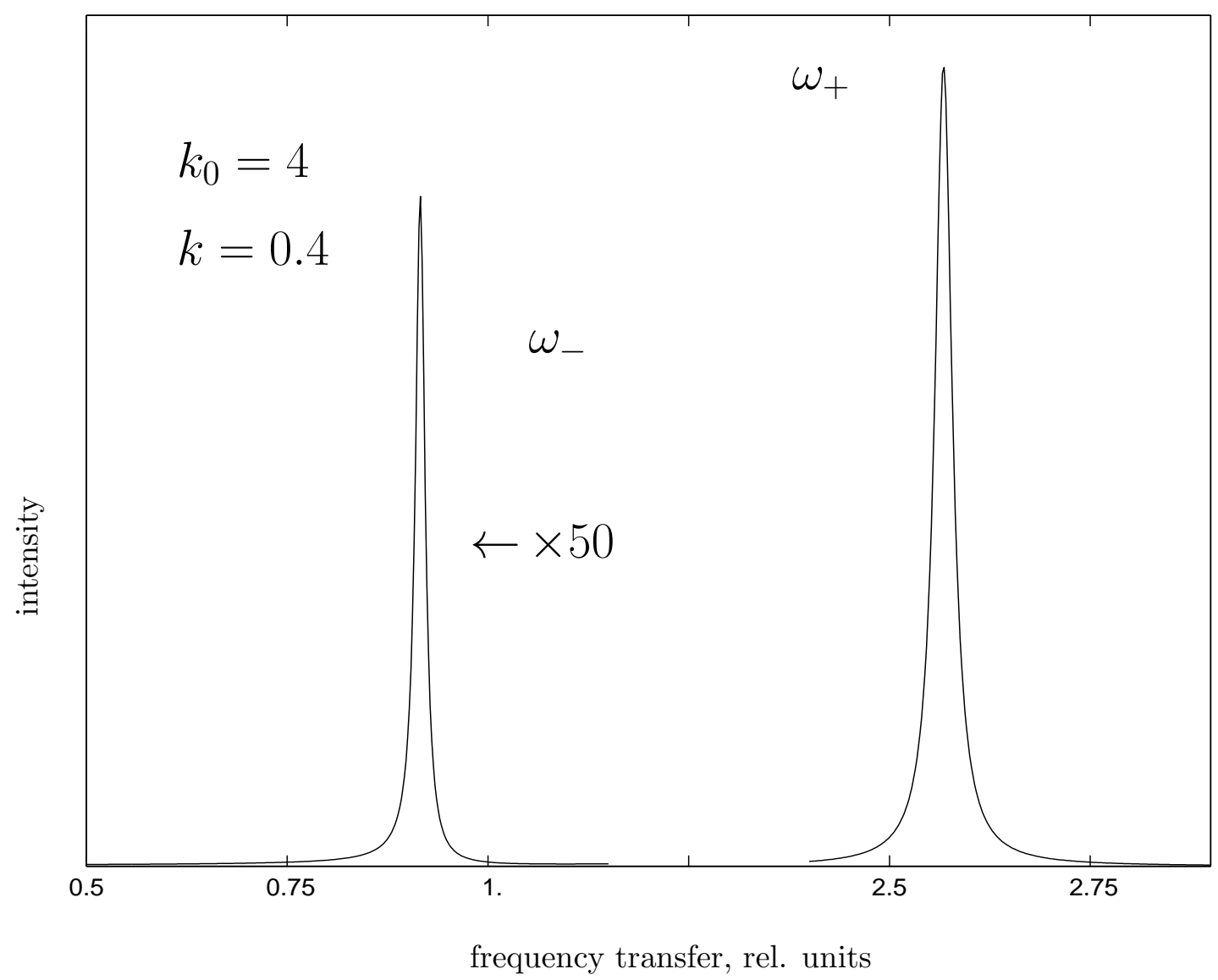

FIG. 3: 




FIG. 4: 


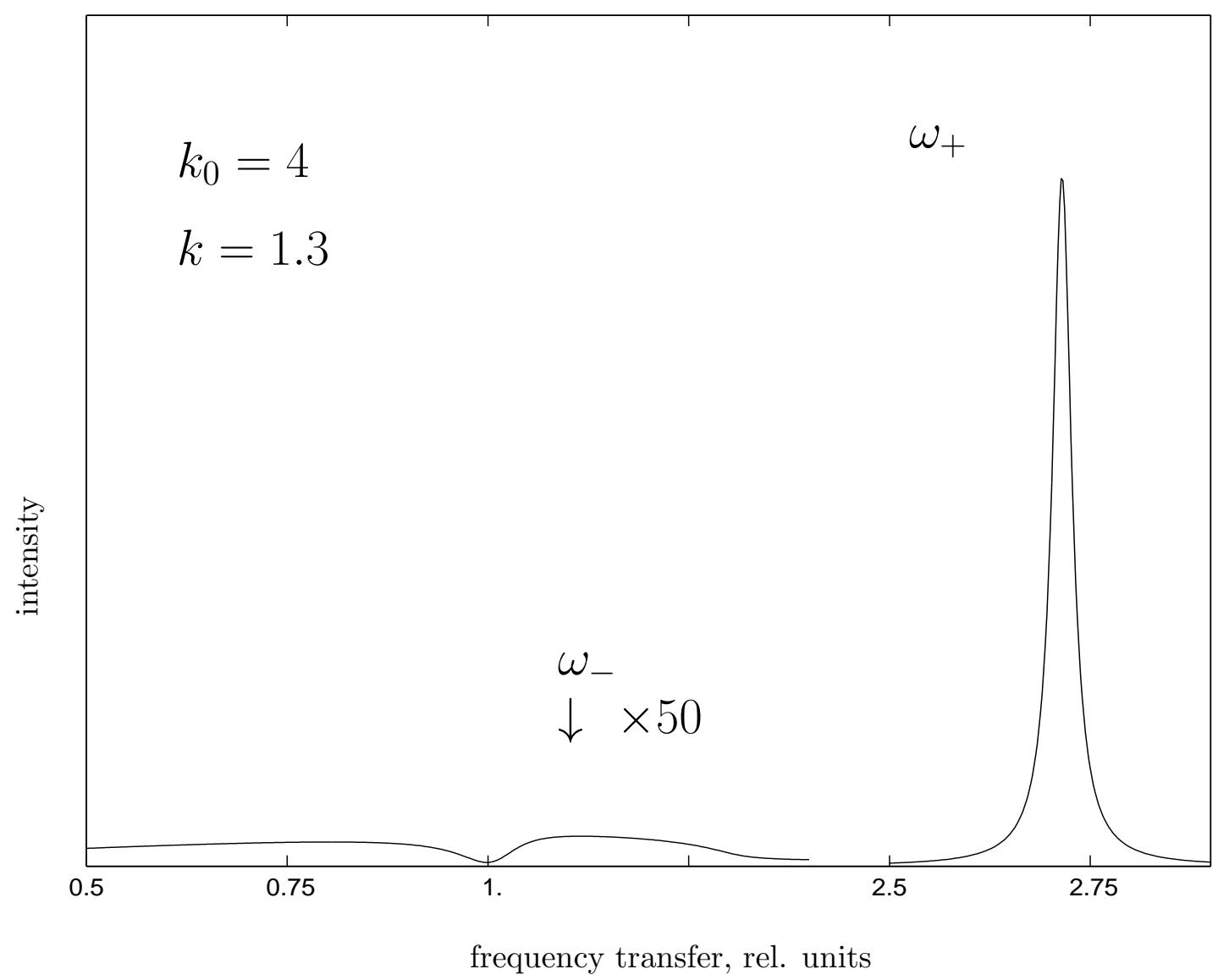

FIG. 5: 


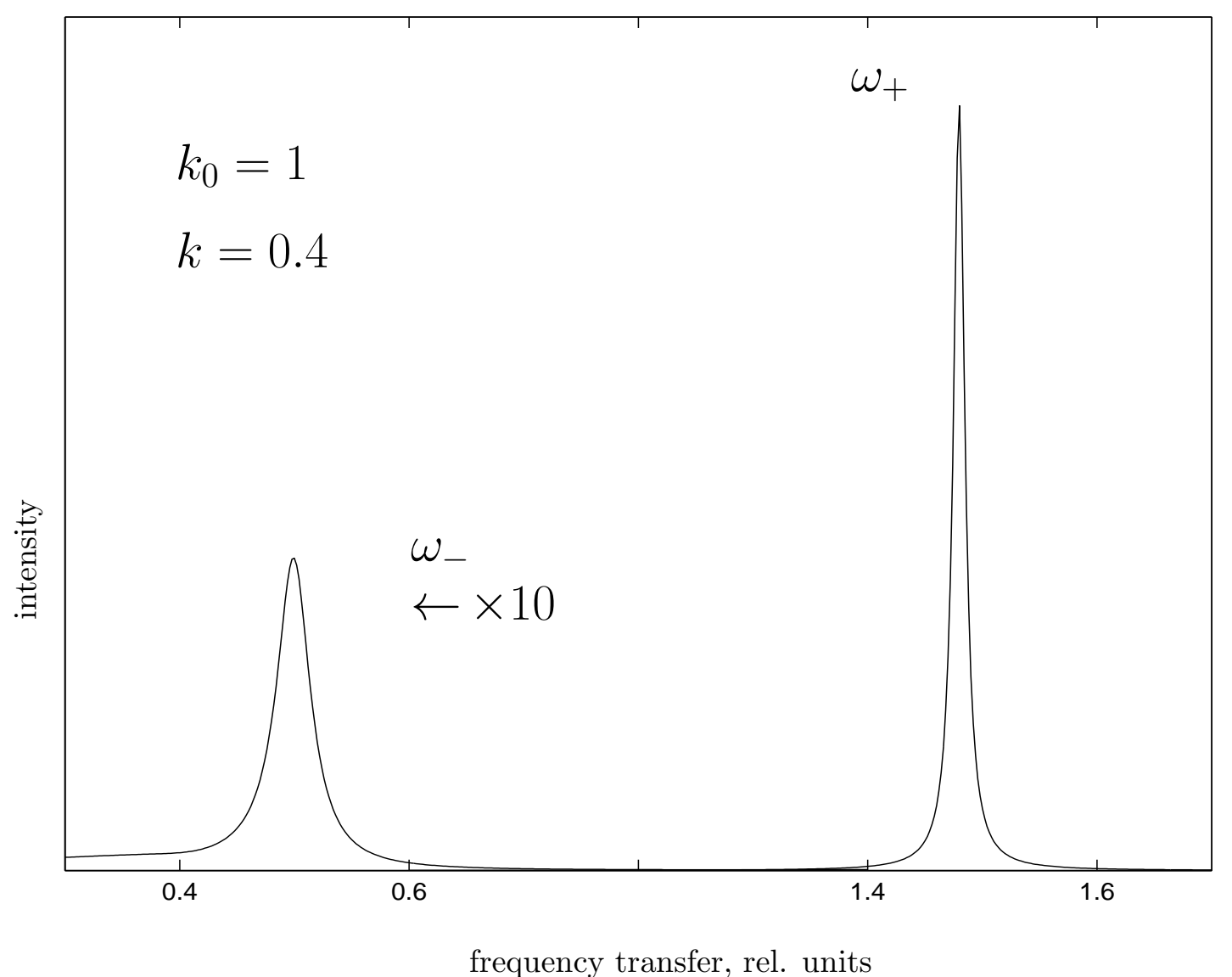

FIG. 6: 


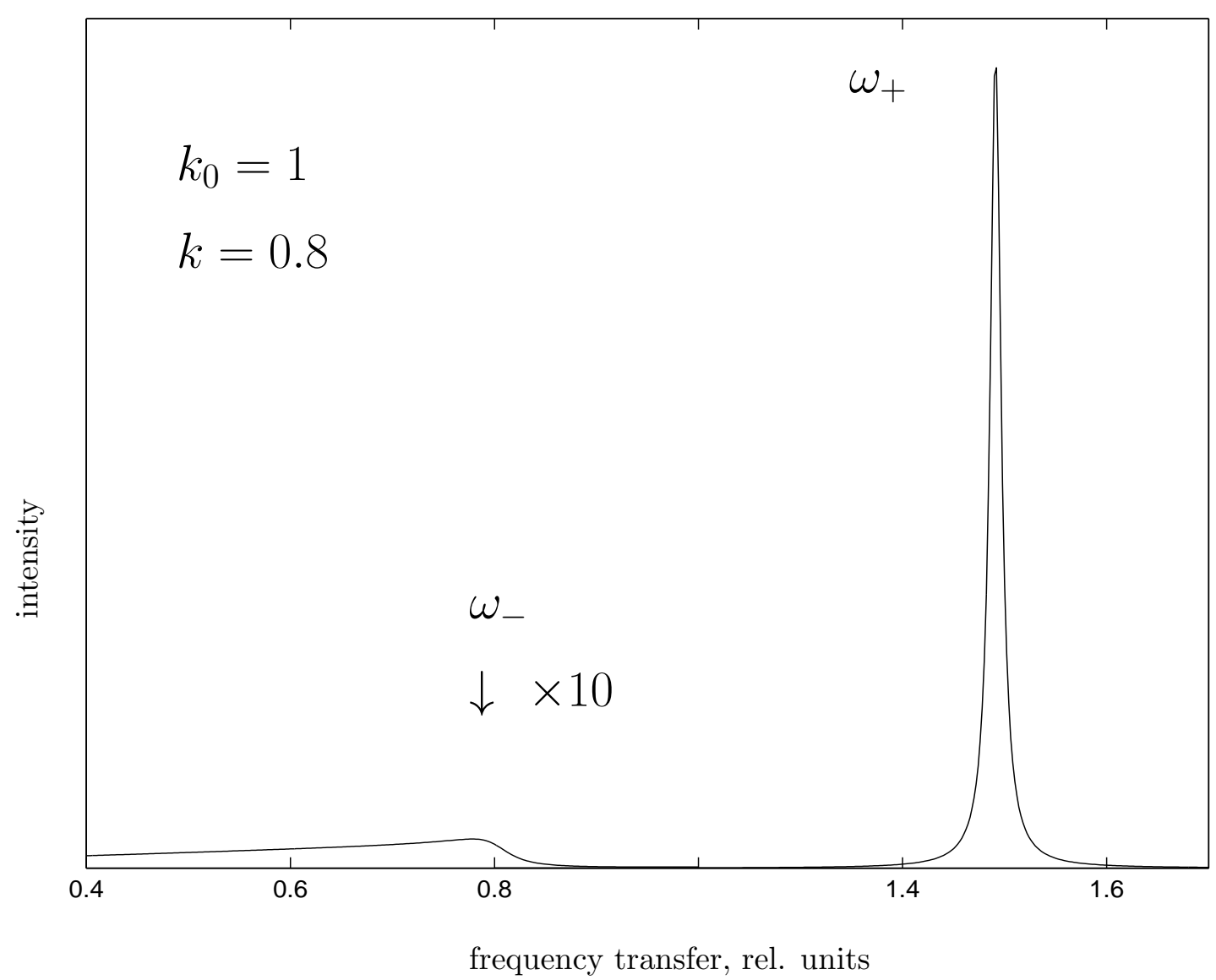

FIG. 7: 


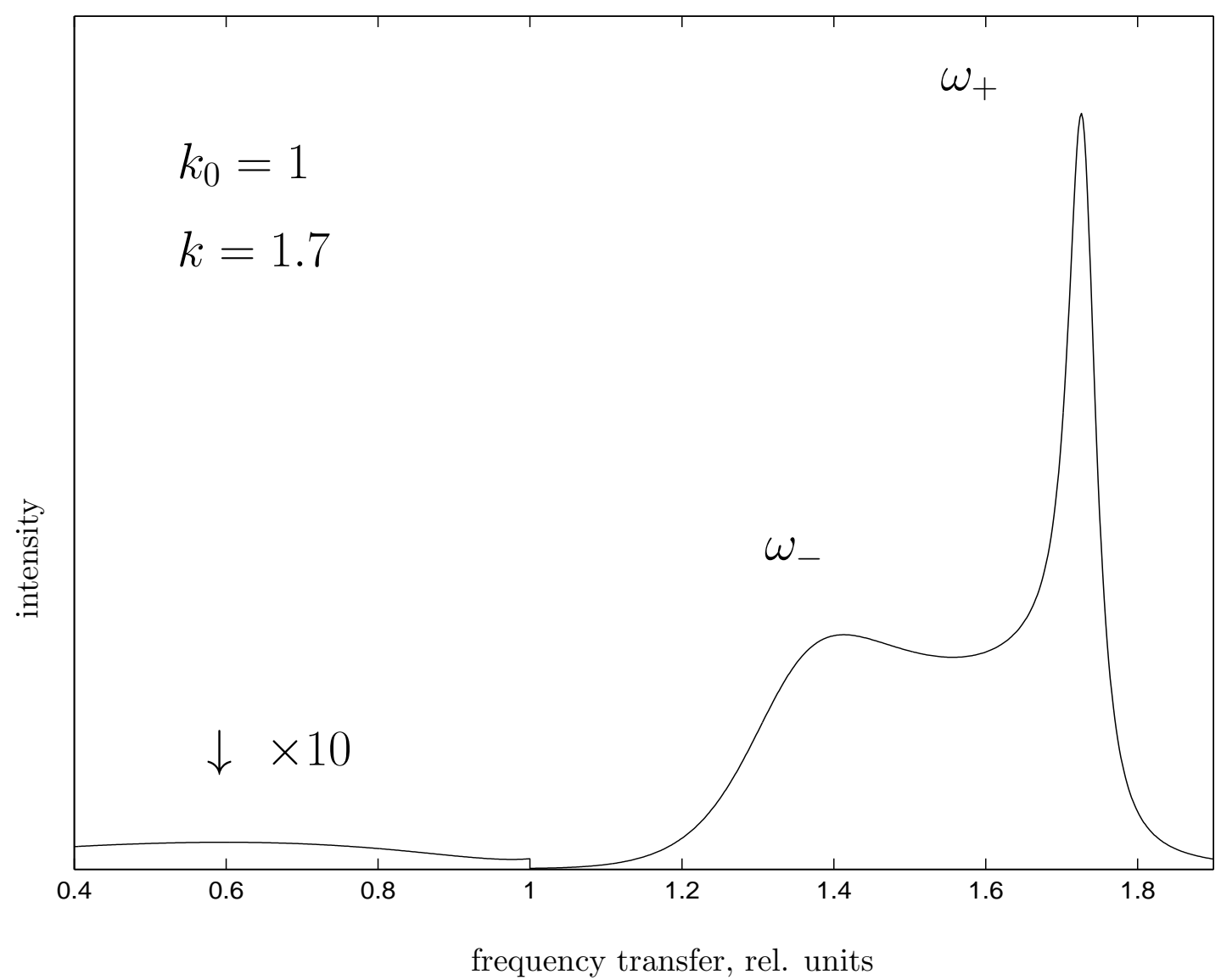

FIG. 8: 